\title{
Interaction of Molecules of Phosphorus-Containing Inorganic Additives with Iron Surface: Quantum-Chemical Analysis and Tribotechnical Testing
}

\author{
Yuri F. Migal ${ }^{1}$, Vladimir I. Kolesnikov ${ }^{2}$, Maria A. Savenkova ${ }^{2}$, Daria N. Solodovnikova² \\ ${ }^{1}$ Southern Scientific Centre of Russian Academy of Sciences, Rostov-on-Don, Russia \\ ${ }^{2}$ Rostov State Transport University, Rostov-on-Don, Russia \\ Email: ymigal@mail.ru
}

Received July 24, 2013; revised August 29, 2013; accepted September 15, 2013

Copyright (C) 2013 Yuri F. Migal et al. This is an open access article distributed under the Creative Commons Attribution License, which permits unrestricted use, distribution, and reproduction in any medium, provided the original work is properly cited.

\begin{abstract}
Condensed phosphates of metaphosphatic structure with the equal relation of metal oxide and phosphorus pentoxide are promising inorganic oleophilic antifrictional, antiwear and anti-seize, heat-resistant and eco-friendly additives to greases. We study in detail one group of these compounds, phosphorowolframates of alkaline metals $\mathrm{M}^{\mathrm{I}} \mathrm{PWO}_{6}\left(\mathrm{M}^{\mathrm{I}}=\mathrm{Li}\right.$, $\mathrm{Na}, \mathrm{K}, \mathrm{Rb}, \mathrm{Cs}$ ), by using the ADF software package based on the DFT approach. Three stages of interaction of molecules of these substances with an iron surface are investigated: change of the shape of molecules near a surface, formation of the adsorptive complex and its disintegration. It is shown that a strong covalent binding between molecules of phosphorowolframates and a surface appears in the course of adsorption. High strength of this binding and also low inner strength of the chain and its flexibility provide effective protection of iron surface against wear. It is also found that at adsorption of phosphorowolframates on an iron surface, there is a change of local structure of the surface.
\end{abstract}

Keywords: Phosphorowolframates of Alkaline Metals; Quantum-Chemical Analysis; Adsorptive Complex; Wear Resistance

\section{Introduction}

Various organic compounds are traditionally used as antiwear and antiscoring additives to plastic lubricants. In the case of hard operating conditions, molecules of these compounds disintegrate as a result of thermooxidizing destruction, causing diverse chemical reactions in base oil. Products of decomposition worsen operational characteristics of greases, provoking corrosion activity, toxic emissions and environmental pollution. Such phenomena are resulting in big considerable economic losses.

The stated negative processes can be prevented by changing the chemical nature of additives so as to eliminate active particles (ions, radicals) which initiate destructive reactions in lubricants at operation. We suppose that condensed phosphates of metaphosphatic structure with the equal relation of metal oxide and phosphorus pentoxide can be promising inorganic oleophilic antifrictional, antiwear and anti-seize, heat-resistant and ecofriendly additives. In particular, phosphoromolybdates of alkaline metals and nickel belong to such substances. It is established that the introduction of these substances into lubricants improves tribotechnical characteristics of greases and enables to converse rheological and operational properties for a long time $[1,2]$.

Similarity of chemical properties of molybdenum and tungsten allows us to assume similarity of properties of their compounds, phosphoromolybdates and phosphorowolframates. Preliminary experiments with phosphorowolframates as additives to lubricants confirm these assumptions. Consequently, it is necessary to study properties of the whole group of similar compounds (i.e. heteropolyphosphates) with a view to search the most effective part of them. Within this research we assume to consider the following questions: 1) change of structure of molecules of heteropolyphosphates near a metal surface; 2) formation and disintegration of an adsorptive complex arising on a surface; 3 ) influence of this complex on local structure of a surface.

Important characteristics of additives are two quantities connected with each other: energy of adsorption and strength of binding between molecules of additives and a metal surface. These quantities show, in fact, how reliably the covering formed by additives protects metal 
against wear. From this point of view, it is important to estimate strength of the chemical binding arising between molecules of heteropolyphosphates and a surface, and compare it with binding strength in case of other compounds which are traditionally used as additives.

In this work, to study the questions mentioned above, we use the quantum-chemical approach which allows us, as appears from the general reasons, to investigate atomic level mechanisms of processes occurring on sliding surfaces. Methods of calculations appearing in three last decades and based on the approach of the density functional theory (DFT) enable us to carry out a rather detailed analysis of interactions of molecules of additives with a surface. What follows the interaction of phosphorowolframates of alkaline metals $\mathrm{M}^{\mathrm{I}} \mathrm{PWO}_{6}\left(\mathrm{M}^{\mathrm{I}}=\mathrm{Li}\right.$, $\mathrm{Na}, \mathrm{K}, \mathrm{Rb}, \mathrm{Cs}$ ) with an iron surface is considered. First, we give the description of the cluster model and a method of calculation of its main characteristics. Second, the structure of molecules of phosphorowolframates being in a free state, and changes of the structure caused by interaction with an iron surface are considered. Then, the results of calculations of energy of interaction of molecules with a surface are given, and the strength of a covering of a surface by molecules is estimated. In the final part, the results of tribotechnical tests are presented, allowing us to estimate in practice the efficiency of application of phosphorowolframates as additives to lubricants.

\section{Model and Calculation Method}

When studying interaction of molecules of additives with a metal surface it is necessary to take into account that the full quantum-chemical analysis of sharply non-uniform systems, such as molecule + surface, is practically impossible because of a huge number of interatomic interactions in systems. The problem can be solved as follows. From a surface layer of metal one chooses some microscopic volume containing atoms located most close to the adsorbed molecule. These atoms together with the molecule represent a polyatomic cluster which allows us to imitate the adsorption phenomenon. The size of the cluster has to be rather big so that qualitative and quantitative regularities of adsorption to be reproduced probably more precisely. On the other hand, the cluster should not be too big to avoid time excess when calculations of its main characteristics are performed. Thus, the choice of the cluster size is a compromise between two inconsistent requirements, the accuracy of reproduction of the main characteristics of adsorption and the limitation of calculation time.

The practice of quantum-chemical calculations shows that in many cases clusters of rather small sizes enable features of the studied phenomenon in solids to be reproduced on qualitative and semi-quantitative levels (see, e.g., [3]). In [4,5] the analysis of dependence of results of calculation of binding energy of clusters, imitating the phenomenon of grain boundary segregation in steel, on the cluster size was carried out. It was established that the cluster with number of atoms $n-18$ can be considered as the minimum cluster by means of which it is possible to investigate changes of binding energy caused by change of chemical composition of a system.

An alternative to the cluster approach described here is so-called zone approach within which the surface is assumed to be infinite and periodic boundary conditions are used. However, within the zone approach it is very difficult to estimate energy of adsorption of molecules, and it induces us to prefer more evident cluster approach.

The main characteristics of cluster model are interatomic distances and the total energy of interatomic bonds. Software package ADF [6] based on the DFT is used to calculate these quantities. This package enables us to study compounds of transitional metals including hundreds atoms that is especially important for researches of processes on an iron surface. Earlier, by means of this package we carried out the analysis of interatomic interactions on a surface of grains in a polycrystalline iron [7]. As a result we have received estimates of compatibility of various elements with iron on the grain surfaces agreeing with known experimental data. This fact allows us to hope that the analysis of interaction of molecules of additives with an iron surface, carried out by means of the ADF package, also yields sufficiently reliable results.

\section{Structure of Molecules of Phosphorowolframates in Free State}

To clarify details of interaction of phosphorowolframates with an iron surface it is necessary to identify structure of initial molecules of these compounds, received by melting, and possible changes of this structure near a surface. We carried out quantum-chemical calculations of compounds $\mathrm{M}^{\mathrm{I}} \mathrm{PWO}_{6}$, where $\mathrm{M}^{\mathrm{I}}$ is an alkaline metal $(\mathrm{Li}, \mathrm{Na}$, $\mathrm{K}, \mathrm{Rb}$ or $\mathrm{Cs}$ ). Possibility of application of the ADF package for such objects was established previously from the example of well studied inorganic molecules containing atoms of phosphorus and tungsten $\mathrm{P}_{4} \mathrm{O}_{6}, \mathrm{P}_{4} \mathrm{O}_{6} \mathrm{~S}_{4}$, $\mathrm{W}(\mathrm{CO})_{6}$. The obtained values of interatomic distances in these molecules practically coincide with experimental data that allows us to consider this method as sufficiently exact for studied compounds.

For calculation of characteristics of phosphorowolframates the basis $\mathrm{TZ2}$ P and an option with the frozen core states (option Small) were used, binding energy was determined within the LDA approach (see [6]). As follows from calculations, molecules of all considered compounds being in a free state have a cyclic form (this fact is confirmed by the infrared spectroscopy method). The molecule of lithium phosphorowolframate has three 
groups $\mathrm{LiPWO}_{6}$, and molecules of other compounds have four groups $\mathrm{M}^{\mathrm{I}} \mathrm{PWO}_{6}$ each. Figure 1 shows some possible forms of lithium and sodium phosphorowolframate molecules with the various point symmetries. Small red circles correspond to oxygen atoms, averages (yellow) to phosphorus atoms, larger (green) to tungsten atoms, and the largest to atoms of an alkaline metal. Atoms of phosphorus and tungsten, making up the closed internal chain, have an environment (close to tetrahedral) from four oxygen atoms. The connection between $\mathrm{P}$ and $\mathrm{W}$ atoms is formed by oxygen bridge atoms. The atoms of alkaline elements, being on the periphery of molecules, are located symmetrically in relation to the atoms of oxygen entering into an environment of $\mathrm{P}$ atoms. Structures of sodium, potassium, rubidium and caesium phosphorowolframates are almost identical, they differ only in $\mathrm{M}^{\mathrm{I}}-\mathrm{O}$ distance between atom of alkaline metal and the closest to it oxygen atoms.

The data regarding the binding energy and structure of the compounds received in calculations are represented in Table 1. It should be noted that binding energy $E_{\mathrm{b}}$ of molecules in the DFT approach can differ considerably from values of the energy determined experimentally. To illustrate the said one can compare the binding energy of the $\mathrm{O}_{2}$ molecule, calculated with the basis used by us and equal to $9.6 \mathrm{eV}$, with experimentally measured energy of dissociation of this molecule equal to $5.08 \mathrm{eV}$. Obviously, the calculated value is approximately $90 \%$ as many as the experimental value. Therefore, strictly speaking, values of $E_{\mathrm{b}}$ should be used only for comparative estimates of characteristics of the similar structures calculated within the same approach.

\section{Structure of Molecules of Phophorowolframates near Iron Surface}

Due to interaction with an iron surface the form of molecules of heteropolyphosphates can significantly change. By the infrared spectroscopy method it was fixed that in the friction process the form of molecules of phosphoromolybdates is transformed from cyclic to linear. This fact was confirmed and explained by means of quantum-chemical calculations. In [2] it was shown that change of a form of molecules is caused by two reasons: 1) by the appearance of a negative electric charge in molecules (that is provided by the transfer of electrons from an iron surface) and 2) by the presence of oxygen in an environment. In a case of phosphoromolybdates there goes a reaction

$$
\mathrm{M}_{\mathrm{n}}^{\mathrm{I}} \mathrm{P}_{\mathrm{n}} \mathrm{Mo}_{\mathrm{n}} \mathrm{O}_{6 \mathrm{n}}+\mathrm{me}+1 / 2 \mathrm{O}_{2} \rightarrow\left(\mathrm{M}_{\mathrm{n}}^{\mathrm{I}} \mathrm{P}_{\mathrm{n}} \mathrm{Mo}_{\mathrm{n}} \mathrm{O}_{6 \mathrm{n}+1}\right)^{\mathrm{m}-}
$$

where $n=3$ (in case of $M^{\mathrm{I}}=\mathrm{Li}$ ) or 4 (in case of $\mathrm{M}^{\mathrm{I}}=\mathrm{Na}$, $\mathrm{K}, \mathrm{Rb}, \mathrm{Cs}$ ), and $\mathrm{m}$ is the number of electrons captured by a molecule.

Similar processes take place also in the case of phosphorowolframates. Linear forms of the lithium phosphorowolframate $\mathrm{Li}_{3} \mathrm{P}_{3} \mathrm{~W}_{3} \mathrm{O}_{18}$, appearing near a surface, are depicted in Figure 2. In Figure 3 dependence of binding energy of cyclic and linear forms of this compound on the charge of molecules is represented.

From this figure it follows that the binding energy is minimum for a linear form of the molecule $\mathrm{Li}_{3} \mathrm{P}_{3} \mathrm{~W}_{3} \mathrm{O}_{19}$ with the charge $2 \overline{\mathrm{e}}$. It signifies that such a molecule is the stablest, and it becomes prevailing in the course of friction.

Analyzing the received results, it is possible to assume that the form of molecules changes as follows (Figure 4). In the beginning, the cyclic molecule $\mathrm{Li}_{3} \mathrm{P}_{3} \mathrm{~W}_{3} \mathrm{O}_{18}$ gets a negative charge $2 \overline{\mathrm{e}}$ from a surface. Then in one of parts of the bond $\mathrm{P}-\mathrm{O}-\mathrm{W}$ there is a rupture of a chain $(\mathrm{P}-\ldots-\mathrm{O}-\mathrm{W})$, and the atom of oxygen remains connected

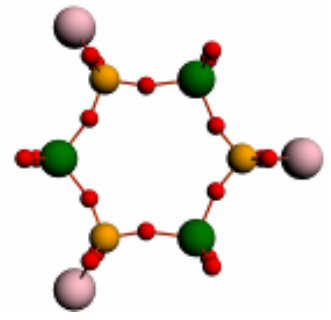

(a)

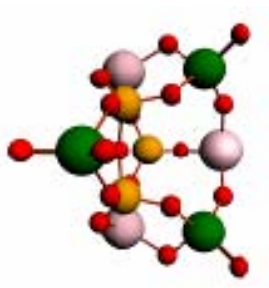

(c)

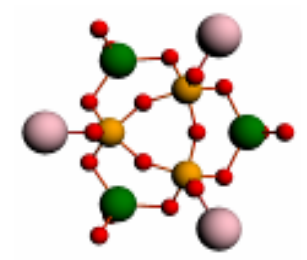

(b)

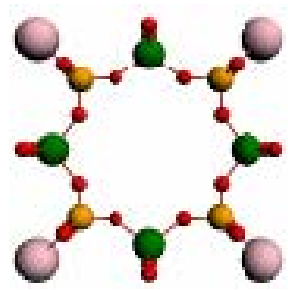

(d)
Figure 1. Three forms of a cyclic molecule of lithium trimetaphosphorowolframate with the point symmetries $D_{3 \mathrm{~h}}(\mathrm{a})$, $\mathrm{C}_{3 \mathrm{v}}(\mathrm{b}), \mathrm{C}_{\mathrm{s}}(\mathrm{c})$, and a cyclic molecule of sodium tetrametaphosphorowolframate with the highest symmetry $D_{4 h}(d)$.

Table 1. Binding energy and geometrical parameters of cyclic molecules calculated for forms with the highest symmetry.

\begin{tabular}{cccccc}
\hline & $\mathrm{LiPWO}_{6}$ & $\mathrm{NaPWO}_{6}$ & $\mathrm{KPWO}_{6}$ & $\mathrm{RbPWO}_{6}$ & $\mathrm{CsPWO}_{6}$ \\
\hline Binding energy, $\mathrm{eV}$ & -214.2 & -282.2 & -283.2 & -283.2 & -284.3 \\
P-O-W distance, $\AA$ & 3.47 & 3.39 & 3.37 & 3.34 & 3.35 \\
M $^{\mathrm{I}}-\mathrm{O}$ distance, $\AA$ & 1.88 & 2.22 & 2.48 & 2.59 & 2.72 \\
\hline
\end{tabular}




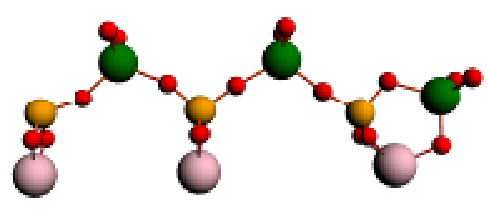

(a)

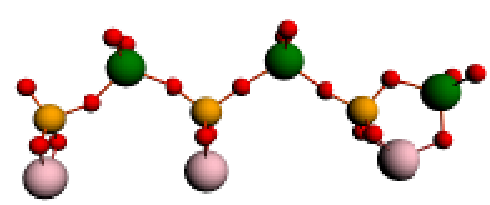

(b)

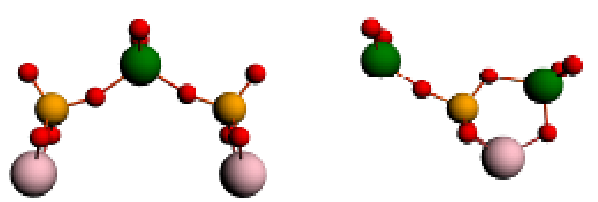

(c)

Figure 2. Linear forms of a molecule of lithium phosphorowolframate $\mathrm{Li}_{3} \mathrm{P}_{3} \mathrm{~W}_{3} \mathrm{O}_{18}$ arising near an iron surface: (a) a radical anion $\left(\mathrm{Li}_{3} \mathrm{P}_{3} \mathrm{~W}_{3} \mathrm{O}_{18}\right)^{2-}$ - with a broken bond at the left end atom of phosphorus; (b) a stable anion chain $\left(\mathrm{Li}_{3} \mathrm{P}_{3} \mathrm{~W}_{3} \mathrm{O}_{19}\right)^{2-}$; (c) the disintegration of the chain into two fragments.

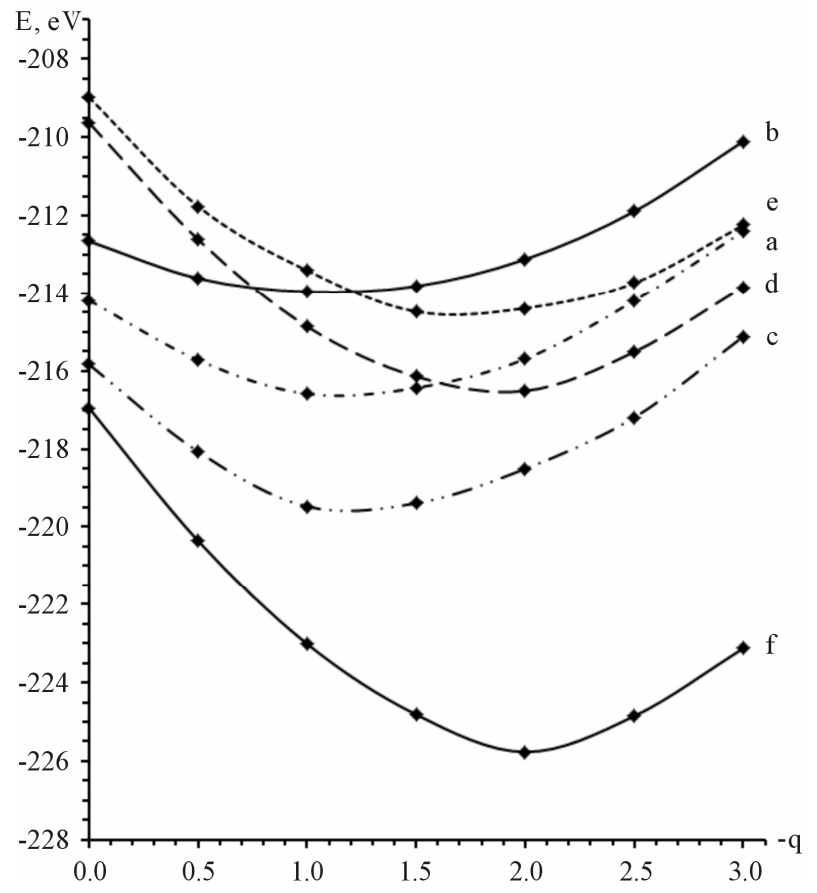

Figure 3. Dependence of the binding energy of cyclic and linear forms of lithium phosphorowolframate $\mathrm{Li}_{3} \mathbf{P}_{3} \mathbf{W}_{3} \mathbf{O}_{18}$ on the charge of molecules: a) a cyclic molecule with the point symmetry $D_{3 h}$; b) a cyclic molecule with the point symmetry $\mathrm{C}_{3 \mathrm{v}}$; c) a cyclic molecule with the point symmetry $\mathrm{C}_{s}$; d) a radical anion $\left(\mathrm{Li}_{3} \mathrm{P}_{3} \mathrm{~W}_{3} \mathrm{O}_{18}\right)^{2-}$ - with a broken bond at the end atom of phosphorus; e) a radical anion $\left(\mathrm{Li}_{3} \mathrm{P}_{3} \mathrm{~W}_{3} \mathrm{O}_{18}\right)^{2-}$ - with a broken bond at the end atom of tungsten; f) a stable anion chain $\mathrm{Li}_{3} \mathrm{P}_{3} \mathrm{~W}_{3} \mathrm{O}_{19}{ }^{2-}$. with atom of tungsten. (The other variant of the rupture, i.e. $\mathrm{P}-\mathrm{O}-\ldots-\mathrm{W}$, as the $\mathrm{O}$ atom remains connected with phosphorus atom is energetically less favorable, but in the course of friction this variant is also possible.)

The molecule, getting a linear form, turns into radical anion $\left(\mathrm{Li}_{3} \mathrm{P}_{3} \mathrm{~W}_{3} \mathrm{O}_{18}\right)^{2-}$ - with a broken bond at the end $\mathrm{P}$ atom. Further, interacting with molecular oxygen, the molecule of phosphorowolframate captures one $\mathrm{O}$ atom and turns into a stable linear anion $\mathrm{Li}_{3} \mathrm{P}_{3} \mathrm{~W}_{3} \mathrm{O}_{19}{ }^{2-}$. The process described above actually happens in one stage because the existence of radical anion is energetically disadvantageous. In all this process the quantity of energy $9.9 \mathrm{\jmath B}$ is given off. This is enough in order that a molecule of oxygen is disintegrated and a reaction similar to reaction (1) is possible. (We should remind that the value of binding energy in the $\mathrm{O}_{2}$ molecule calculated in the approach used by us is equal to $9.6 \mathrm{eV}$, and according to Equation (1), for course of the reaction only a half of this value is necessary.)

The chain $\mathrm{Li}_{3} \mathrm{P}_{3} \mathrm{~W}_{3} \mathrm{O}_{19}{ }^{2-}$ formed is not so strong. Calculations show that for the disintegration by the way represented in Figure 2(c) it is sufficient to provide the energy equal to $3.2 \mathrm{eV}$.

This energy is significantly less than the energy required, for example, for disintegration of a molecule of oxygen. This fact will be discussed below as the mechanism of protection of an iron surface against wear will be considered.

Let us consider one more important circumstance connected with the usage of phosphorowolframates in lubricants. It is known that the presence of atomic phosphorus as an impurity in iron leads to the deterioration of properties of metal. In this connection, it is of particular interest to examine the possibility of the diffusion of atomic phosphorus from compounds considered by us into an iron volume. For obtaining an approximate value of the energy necessary for the atomization of phosphorus (its isolation from a compound structure) and the diffusive penetration into metal, it is enough to examine the disintegration of a tetrahedral complex $\mathrm{PO}_{4}^{q}$ entering the structure of phosphorowolframates and having an effective charge $q$. It was found that this value is about $30 \mathrm{eV}$. Thus, the probability of the penetration of atomic phosphorus into an iron volume is rather small under conditions considered.

\section{Adsorption of Molecules of Phosphorowolframates on Surface}

The appearance of a linear form of heteropolyphosphates of alkaline metals on an iron surface is essentially important for applicability of these compounds as additives to greases. As their concentration becomes large, the molecular chains are arranged perpendicularly to a surface. The external ends of the chains, interacting with 


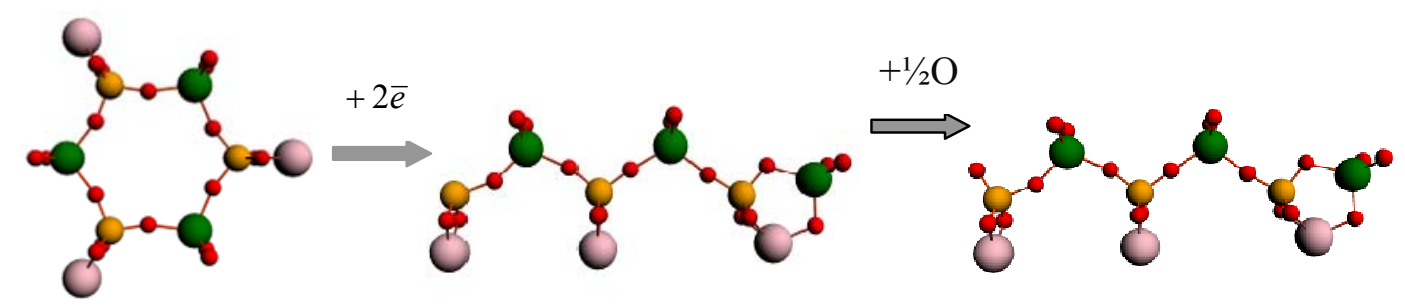

Figure 4. Change of a form of a molecule of lithium phosphorowolframate $\mathrm{Li}_{3} \mathrm{P}_{3} \mathrm{~W}_{3} \mathrm{O}_{18}$ near an iron surface.

greasing, provide, finally, a smooth sliding of a counterbody along an iron surface with a small coefficient of friction.

The adsorption of molecules of phosphorowolframates on a juvenile iron surface is considered below. Such a surface can be formed on the most coming out roughness where the continuity of the oxidic layer covering metal can be broken in the course of friction.

As a model of a juvenile surface we choose the cluster from 13 iron atoms which reflects properties of a real object with sufficient accuracy. Owing to the technical reasons hampering calculation of adsorption of the whole chain of a phosphorowolframate, we considered interaction of an iron cluster only with end fragments of a chain, phosphatic and wolframatic (Figure 5).

For the coordination of fragments shown in Figure 5, we suppose that the strongest binding of a chain with a metal surface (100) arises through two atoms of oxygen, each of which is connected with two iron atoms. Possibility of such a binding is obvious for the wolframatic end, and in case of the phosphatic end it is possible to expect that in the course of adsorption the lithium atom from an environment of phosphorus gets to be connected only with one of atoms of oxygen, and the second becomes free to connect with iron.

In our opinion, the model represented in Figure 5 can be suitable also in case of adsorption on a surface covered with an oxide film. It is quite admissible that the chain of radical anion $\left(\mathrm{Li}_{3} \mathrm{P}_{3} \mathrm{~W}_{3} \mathrm{O}_{18}\right)^{2-}$ - can not capture an atom of oxygen from the lubricant environment, and get it being built into a film. Thus we receive the configuration represented in Figure 5 again.

The main question arising from such modeling is the extent of error caused by rejection of the rest of a chain. As energy of adsorption is determined on the whole by the distribution of the electronic density at the place of the contact of adsorbent and adsorbate, in this case it should be considered how the electronic density within the end fragment can change if this fragment is separated from a chain. For accounting all possible variants of the distribution of electronic density, a varied parameter, an effective charge $q$ of the fragment is introduced and a wide range of its values (from 0 to -3 ) is examined. Since the iron cluster is assumed not to be loaded prior to adsorption, the general charge of the adsorptive complex

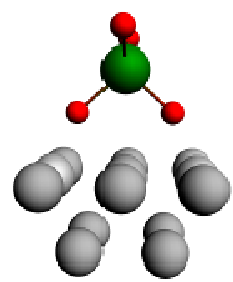

Figure 5. Interaction of end fragments $\mathrm{PO}_{4}^{q-}$ or $\quad \mathrm{WO}_{4}^{q-}$ with a cluster modeling an iron surface.

is also accepted to be equal to $q$.

Figure 5 shows just schematically the structure of the adsorptive complex consisting of a cluster of iron and the end fragment of a chain. The variation of parameters of the complex, that is necessary for discovering the state with the minimum energy, showed that in the course of adsorption there are essential changes of the arrangement of atoms in an iron cluster (these changes are reflected in Figure 6). Two Fe atoms closest to a molecule move to it, and other atoms rearrange. In the course of adsorption there is also a redistribution of atomic charges, depending on the $q$ value. As a result, there arises a rather strong covalent binding between the chain and the cluster.

To study the mechanism of surface wear it is necessary to consider not only process of the formation of the adsorptive complex, but also process of its disintegration. In accord with calculations the disintegration of the adsorptive complex $\mathrm{Fe}_{13} \mathrm{XO}_{4}(\mathrm{X}=\mathrm{P}, \mathrm{W})$ caused by a power action can occur in two ways represented in Figure 6. The first of them is the division into initial parts $\mathrm{Fe}_{13}$ and $\mathrm{XO}_{4}$, with absorption of energy $E_{1}$, and the second is the disintegration with ejection of two iron atoms from a cluster, i.e. the division into $\mathrm{Fe}_{11}$ and $\mathrm{Fe}_{2} \mathrm{XO}_{4}$, with absorption of energy $E_{2}$.

Energy of formation and disintegration of the adsorptive complex is calculated by using equation $E_{\mathrm{ad}}=E_{\text {tot }}$ $-E_{\mathrm{Fe}}-E_{\mathrm{PW}}$, where $E_{\mathrm{tot}}$ is the energy of an integrated cluster including iron atoms and the end fragment of a chain, $E_{\mathrm{Fe}}$ is the energy of a cluster of iron containing 13 or 11 atoms, $E_{\mathrm{PW}}$ is the energy of the fragment of a chain (with addition of two $\mathrm{Fe}$ atoms at calculation of $E_{2}$ ). The dependence of energies $E_{1}$ and $E_{2}$ on the total charge $q$ is represented in Tables 2 and 3. It is obvious from the figures and tables that $E_{2}<E_{1}$. Thus, it signifies that the variant of disintegration of the adsorptive complex with 


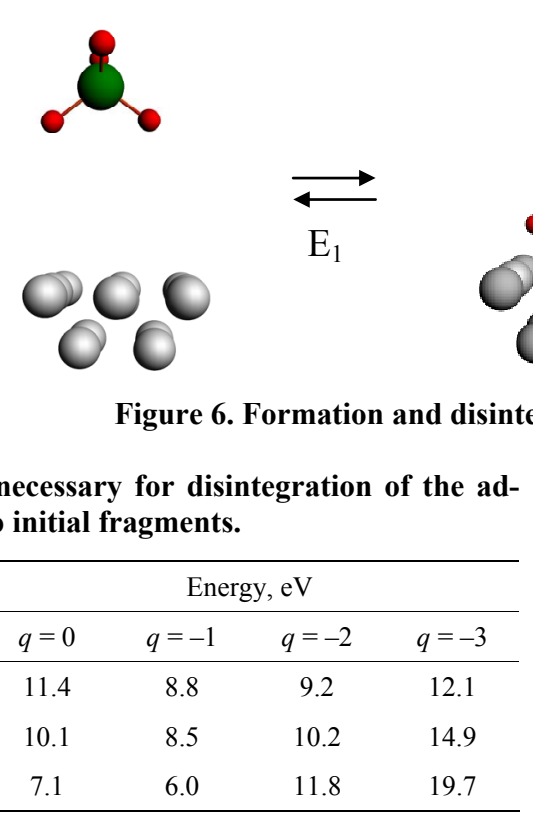

Table 3. Energy $E_{2}$ necessary for disintegration of the adsorptive complex with ejection of iron atoms.

\begin{tabular}{ccccc}
\hline \multirow{2}{*}{ Molecular fragment } & \multicolumn{4}{c}{ Energy, eV } \\
\cline { 2 - 5 } & $q=0$ & $q=-1$ & $q=-2$ & $q=-3$ \\
\hline $\mathrm{WO}_{4}^{q}$ & 6.2 & 5.8 & 8.3 & 11.0 \\
$\mathrm{PO}_{4}^{q}$ & 6.0 & 5.9 & 7.5 & 10.7 \\
$\mathrm{COOH}^{q}$ & 6.3 & 7.1 & 9.8 & 13.1 \\
\hline
\end{tabular}

the ejection of $\mathrm{Fe}$ atoms is more probable.

It follows from Tables $\mathbf{2}$ and $\mathbf{3}$ that the values of energy necessary for disintegration of the adsorptive complex with the participation of phosphatic and wolframatic fragments are rather close to each other. Intervals of these values for different $q$ are overlapping. For this reason, there are no bases to consider that binding of a chain with an iron surface through any fragment is stronger than through the other fragment. It is not excluded that, in the course of friction, phosphorowolframates dissolved in a lubricant can be attached to iron surface through both ends equiprobably.

To estimate the efficiency of phosphorowolframates as additives to lubricants it is also necessary to compare the strength of the binding arising between phosphorowolframates and an iron surface with the binding strength in case of other compounds which are traditionally applied as additives. To this end, we calculated the energy of adsorption of the highest carbonic acids simulated in this work by the end fragment $\mathrm{COOH}$. The data presented in Tables 2 and 3 allow us to state that phosphorowolframates are attached to iron surface more strongly than the highest carbonic acids. Besides, at a power action on the adsorbed layer from molecules of the highest carbonic acids, the separation of these molecules together with iron atoms is less probable, than in case of phosphorowolframates. This fact also testifies to less strong binding of the highest carbonic acids with an iron surface.

A qualitative estimation of the strength of the binding of the studied compounds with an iron surface can be carried out also by means of quantities based on the DFT approach: electronic chemical potential $\mu$ and chemical hardness $\eta$ (see [8,9]). As two subsystems A and B are united, the strength of arising binding can be estimated by means of a ratio

$$
\Delta N \approx \frac{\mu_{B}-\mu_{A}}{2\left(\eta_{A}+\eta_{B}\right)},
$$

where $\Delta N$ is the quantity of transferred electrons,

$$
\begin{gathered}
\mu=-\left(\varepsilon_{\text {LUMO }}+\varepsilon_{\text {НОМО }}\right) / 2, \\
\eta=\left(\varepsilon_{\text {LUMO }}-\varepsilon_{\text {HОМO }}\right) / 2 .
\end{gathered}
$$

Calculations performed by using these equations yield the following results. At formation of the binding $\mathrm{Fe}_{13}+$ $\mathrm{Li}_{3} \mathrm{P}_{3} \mathrm{~W}_{3} \mathrm{O}_{19}{ }^{2-}$ the $\Delta N$ value is equal to 2.8 , and in case of $\mathrm{Fe}_{13}+\mathrm{HCOOH}$ the $\Delta N$ value is -0.1 . This result is one more confirmation of higher strength of binding of phosphorowolframates with an iron surface.

The obtained data relating to the properties of phosphorowolframates enable us to consider the possibility of using these compounds to protect an iron surface against wear. High strength of binding between a surface and the adsorbed molecules of phosphorowolframates (or their fragments) provides long-life covering of a surface. The linear form of molecules provides their high flexibility thanks to what at low mechanical loadings the adsorbed layer is easily deformed, but not destroyed, and at loading removal it is quickly restored. At high loadings, linear chains of molecules are destroyed due to a low intramolecular binding strength, brought energy is absorbed within the adsorbed layer, without affecting a covering from the fragments being immediately on an iron surface. At loading removal, molecular chains can be restored, and the adsorbed layer can also return to the initial state. (Such a restoration won't happen if a power action is great enough for fragments of chains to get de- 
stroyed or for separation of fragments of molecules together with $\mathrm{Fe}$ atoms from a surface.)

\section{Tribotechnical Testing of Phosphorowolframates}

Testing of phosphorowolframates as additives were carried out as follows. Various quantities of these substances were introduced into lubricants Puma and Buksol which are widely used on the railroads of Russia. These mixtures were put on the steel $65 \mathrm{G}$ whose chemical composition is similar to the composition of wheel and rail steels. The estimation of wear resistance of the material was carried out by means of a four-ball friction testing machine. Tests were carried out by a standard technique at loading $196 \mathrm{~N}$ and measurement time $60 \mathrm{~min}-$ utes. The diameter of the wear spot was measured by means of a three-dimensional microscope. Values of the diameter in a case of the lithium phosphorowolframate are represented in Tables $\mathbf{4}$ and $\mathbf{5}$.

These results show that due to the introduction of phosphorowolframates into lubricants the diameter of the wear spot significantly decreases (by an average of 35\%). In photos of sliding surfaces after testing there are smoothed microreliefs with a regular leveled structure. Such a structure provides a larger area of contact between bodies at a triboconjugation, reduces local pressure and promotes the uniform distribution of loadings.

\section{Conclusions}

The performed quantum-chemical analysis shows that at adsorption of phosphorowolframates of alkaline metals on an iron surface, there is a change of local structure of a surface. There raises a rather strong covalent binding between molecules of phosphorowolframates and the iron surface. The strength of this binding is significantly

Table 4. Results of tribotechnical testing of additives introduced into Puma compositions (MP, MG and ML).

\begin{tabular}{|c|c|c|c|c|}
\hline \multirow{3}{*}{ Additive } & \multirow{3}{*}{$\begin{array}{l}\text { Content of additive in } \\
\text { lubricant, } \% \text { by mass }\end{array}$} & \multicolumn{3}{|c|}{ Lubricant composition } \\
\hline & & MP & MG & ML \\
\hline & & \multicolumn{3}{|c|}{ Diameter of wear spot, $\mathrm{mm}$} \\
\hline \multirow{3}{*}{$\mathrm{LiPWO}_{6}$} & 0 & 0.69 & 0.68 & 0.64 \\
\hline & 1 & 0.51 & 0.49 & 0.48 \\
\hline & 5 & 0.58 & 0.53 & 0.51 \\
\hline
\end{tabular}

Table 5. Results of tribotechnical testing of additives introduced into Buksol lubricant.

\begin{tabular}{ccc}
\hline Additive & $\begin{array}{c}\text { Content of additive in } \\
\text { lubricant, \% by mass }\end{array}$ & $\begin{array}{c}\text { Diameter of wear } \\
\text { spot, mm }\end{array}$ \\
\hline $\mathrm{LiPWO}_{6}$ & 0 & 0.51 \\
& 1 & 0.41 \\
\hline
\end{tabular}

higher than at adsorption of the highest carbonic acids which are traditionally applied as additives to lubricants. Intensive power action on the adsorbed layer consisting of molecules of phosphorowolframates can cause separation of these molecules together with iron atoms.

A high strength of binding between molecules of phosphorowolframates and an iron surface, on the one hand, and a low strength of the chains themselves and their flexibility, on the other hand, provide effective protection of an iron surface against wear.

The performed tribotechnical testing of lubricant compositions Puma and Buksol with phosphorowolframates introduced into their structure confirm the efficiency of such additives.

\section{Acknowledgements}

This work was supported by grants from the Russian Foundation for Basic Research, projects 11-08-00640 and 13-08-00732.

\section{REFERENCES}

[1] V. I. Kolesnikov, M. A. Savenkova, S. B. Bulgarevich, A. V. Chelokhyan, E. A. Bulavina and M. V. Boiko, "Investigation of Triboengineering Characteristics of Plastic Railway Greases with Inorganic Polymeric Additives," Journal of Friction and Wear, Vol. 29, No. 3, 2008, pp. 200267. http://dx.doi.org/10.3103/S1068366608030082

[2] V. I. Kolesnikov, Yu. F. Migal, M. A. Savenkova and S. N. Mizhiritskaya, "Adsorption of Heteropolyphosphates of Alkaline Metals on Iron Surface," Journal of Friction and Wear, Vol. 30, No. 6, 2009, pp. 404-410.

http://dx.doi.org/10.3103/S1068366609060051

[3] M. V. Lebedev, "Mechanism of $\mathrm{H}_{2} \mathrm{~S}$ Molecule Adsorption on the GaAs(100) Surface: Ab Initio QuantumChemical Analysis," Physics of the Solid State, Vol. 48, No. 1, 2006, pp. 164-171. http://dx.doi.org/10.1134/S1063783406010306

[4] V. I. Kolesnikov, Yu. F. Migal, S. N. Mizhiritskaya and V. N. Doronkin, "Quantum-Chemical Investigation of the Effect of Grain Boundary Segregation on Wear Resistance of Steel," Journal of Friction and Wear, Vol. 29, No. 2, 2008, pp. 99-107.

http://dx.doi.org/10.3103/S1068366608020049

[5] V. I. Kolesnikov, A. T. Kozakov and Yu. F. Migal, "Study of Friction and Wear in the Wheel-Rail System by XRay Electron and Auger-Electron Spectroscopy and Quantum chemistry," Journal of Friction and Wear, Vol. 31, No. 1, 2010, pp. 11-22. http://dx.doi.org/10.3103/S1068366610010022

[6] G. te Velde, F. M. Bickelhaupt, S. J. A. van Gisbergen, C. Fonseca Guerra, E. J. Baerends, J. G. Snijders and T. Ziegler, "Chemistry with ADF," Journal of Computational Chemistry, Vol. 22, No. 9, 2001, pp. 931-967. http://dx.doi.org/10.1002/jcc.1056

[7] Yu. F. Migal, V. I. Kolesnikov, V. N. Doronkin and E. S. Novikov, "Interaction of Atoms with Grain Surfaces in 
Steel: Periodic Dependence of Binding Energy on Atomic Number and Influence on Wear Resistance," Advances in Materials Physics and Chemistry, Vol. 2, 2012, pp. 201207. http://dx.doi.org/10.4236/ampc.2012.24030

[8] R. G. Pearson, "Absolute Electronegativity and Hardness Correlated with Molecular Orbital Theory," Proceedings of the National Academy of Sciences, Vol. 83, No. 22,
1986, pp. 8440-8441.

http://dx.doi.org/10.1073/pnas.83.22.8440

[9] S. H. Li, H. Yang and Y. S. Jin, "Lubrication Chemistry Viewed from DFT-Based Concepts and Electronic Structural Principles," International Journal of Molecular Sciences, Vol. 5, No. 1, 2004, pp. 13-34. 